\title{
Porting Crystal Structure Data to Virtual Reality using CAD2VR Nichole Valdez ${ }^{1}$, Mark Rodriguez ${ }^{2}$, John Krukar ${ }^{3}$, Matthew Gallegos ${ }^{4}$, James Harris ${ }^{5}$ ${ }^{1}$ Sandia National Laboratories ${ }^{2}$ Sandia National Laboratories, ${ }^{3}$ Sandia National Laboratories, ${ }^{4}$ Sandia National Laboratories, ${ }^{5}$ Sandia National Laboratories nrvalde@sandia.gov
}

Three-dimensional crystal structure data is most often displayed on two-dimensional surfaces such as printed images or computer screens. These displays create visualization issues for extended structures like minerals or metal organic frameworks (MOFs) where packing the structure causes many atoms to overlap. With virtual reality hardware becoming more accessible, the next natural step for crystallography is to visualize 3D structures in 3D space.

CAD2VR software was developed at Sandia National Laboratories to visualize Computer Aided Drafting (CAD) Models in virtual reality (VR). It offers a variety of interaction tools that, when applied to the atomic scale, would be useful for crystallographers. A plugin is being developed that can import crystal structure models into VR. Features include measuring atomic distances, bond lengths, and bond angles; displaying the unit cell; and drawing polyhedron volumes to measure void spaces.

Sandia National Laboratories is a multi-mission laboratory managed and operated by National Technology and Engineering Solutions of Sandia, LLC, a wholly owned subsidiary of Honeywell International, Inc. for the U.S. Department of Energy's National Nuclear Security Administration under contract DE-NA0003525.

This paper describes objective technical results and analysis. Any subjective views or opinions that might be expressed in the paper do not necessarily represent the views of the U.S. Department of Energy or the United States Government. 